\title{
On Optimal QoS-aware Physical Carrier Sensing for IEEE 802.11 Based WLANs: Theoretical Analysis and Protocol Design
}

\author{
Yanfeng Zhu, Student member, IEEE, Qian Zhang, Senior member, IEEE, \\ Zhisheng Niu, Senior member, IEEE, and Jing Zhu, Member, IEEE
}

\begin{abstract}
In Wireless Local Area Networks (WLANs), traditional Physical Carrier Sensing (PCS), which aims at eliminating hidden terminals completely, brings too many exposed terminals and degrades the throughput seriously. Some existing work has proven that an aggressive PCS, which turns up the PCS threshold to allow the existence of hidden terminals, can improve the throughput by balancing the tradeoff between hidden terminals and exposed terminals. However, little work has been conducted to compute the optimal PCS threshold. To address this issue, in this paper we develop an analytical model, which can be used to compute the optimal PCS threshold and investigate the impact of the aggressive PCS on the Quality-of-Service (QoS) in terms of the packet loss rate. Then, we propose a QoS-aware aggressive PCS tuning algorithm, with which users can adapt the PCS threshold to the varying network conditions and the QoS requirement. Extensive simulation results show that the proposed algorithm obtains significant throughput gain compared to the traditional PCS and bounds the packet loss rate below the QoS requirement. The unfairness issue brought by the aggressive PCS is also discussed and evaluated with experimental results at the end of the paper.
\end{abstract}

Index Terms-Physical carrier sensing, spatial reuse, Qualityof-Service, throughput.

\section{INTRODUCTION}

$\mathbf{T}$ HE past few years have seen an explosion in the market of IEEE 802.11 [12] based mobile computing devices. IEEE 802.11 based networks can provide mobile users with broadband connectivity to the backbone Internet in various environments such as airport, office, and campus [5]. Most noticeably, IEEE 802.11 wireless technologies provided by the like of Intel Centrino ${ }^{\mathrm{TM}}$ laptops offer the convenience and flexibility that enables the high density deployment of wireless networks.

In IEEE 802.11 based networks with high density deployment, increasing the throughput per user is challenging due to the limitation of Carrier Sensing Multiple Access (CSMA) mechanism employed in IEEE 802.11. With CSMA,

Manuscript received November 17, 2006; revised May 18, 2007; accepted June 28, 2007. The associate editor coordinating the review of this paper and approving it for publication was X. Zhang.

Y. Zhu and Z. Niu are with the Department of Electronic Engineering, Tsinghua University, Beijing P.R. China (e-mail: zhuyf03@mails.tsinghua.edu.cn; niuzhs@tsinghua.edu.cn).

Q. Zhang is with the department of Computer Science, Hong Kong University of Science and Technology (e-mail: qianzh@cs.ust.hk).

J. Zhu is with Intel Corporation, 2111 NE 25th Ave., JF3-206, Hillsboro, OR 97124, USA (e-mail: jing.z.zhu@intel.com).

Digital Object Identifier 10.1109/TWC.2008.060953. a user performs Physical Carrier Sensing (PCS) before a data transmission to sample the energy in the channel. The transmission proceeds only if the sampled energy is below a threshold known as PCS threshold. Therefore, the throughput per user can be approximately given by $C\left(1-P_{L}\right) / n_{c}$, where $C, P_{L}$, and $n_{c}$ refer to the link capacity, the frame loss rate, and the number of users in the PCS range, respectively. Herein, $C$ is constrained by the employed physical technologies and the channel conditions, and thus it is independent of the PCS threshold. Differently, both $P_{L}$ and $n_{c}$ are relevant to the PCS threshold.

Traditional PCS threshold tuning algorithms [2][3][4] aimed at eliminating hidden terminals completely, i.e., $P_{L} \approx 0 .{ }^{1}$ Under this design principle, maximizing the throughput per user is equal to minimizing $n_{c}$. The feasible method is to tune the PCS threshold so that making the PCS range exactly cover the interfering range. However, from theoretical and experimental points of view, [14][16] respectively demonstrated that an aggressive PCS threshold, which is large enough to allow the existence of hidden terminals, can enhance the throughput per user significantly. In particular, the study in [16] illustrated that $P_{L}$ generally increases with the PCS threshold, while $n_{c}$ decreases with the PCS threshold. Therefore, from the viewpoint of maximizing the throughput per user, there is a tradeoff between $P_{L}$ and $n_{c}$, and the key of such a tradeoff is the PCS threshold. From the experimental results in [14], with physical data rate $24 \mathrm{Mbps}$, a proper aggressive PCS threshold can obtain up to $70 \%$ throughput increase compared to that with the default threshold of IEEE 802.11. Although the existing work has pointed out the need for an aggressive PCS, how to obtain an optimal PCS threshold is still an open issue.

Currently, there are two major challenges for optimizing the PCS threshold in practical networks: one is constructing a closed-form expression to describe the relation between the throughput per user and the PCS threshold, and the other is adapting the PCS threshold to the varying network conditions. Given the user density, $n_{c}$ can be described by a closedform expression with respect to the PCS threshold ${ }^{2}$. Then, the essential part left is to construct the relationship between

\footnotetext{
${ }^{1}$ The probability that a collision occurs due to simultaneous transmissions is much smaller than that due to concurrent transmissions of hidden terminals, and thus eliminating the hidden terminals results in $P_{L} \approx 0$.

${ }^{2} n_{c}$ equals the product of the user density and the area of carrier sensing range.
} 
$P_{L}$ and the PCS threshold, which has been demonstrated to be very difficult in [16]. On the other hand, in practical networks, the optimal PCS threshold in fact dynamically varies with the varying network conditions (such as the user density and the transmission probability of each user) which are tough to be estimated on-line. In addition, the aggressive PCS seriously affects the Quality-of-Service (QoS), which is generally measured in terms of the delay and packet loss rate $^{3}$. The delay decreases with the MAC throughput, and thus it is improved by the aggressive PCS. However, IEEE 802.11 specifies that a data packet will be dropped after several retransmission attempts in MAC layer, and thus a larger $P_{L}$ will result in higher packet loss rate. To address this issue, $P_{L}$ needs to be bounded in MAC layer while enhancing the throughput.

Targeting at the challenges described above, in this paper we develop an analytical model to investigate the relation among the throughput per user, the packet loss rate and the PCS threshold, and then based on the analytical model, we propose a QoS-aware heuristic algorithm to tune the PCS threshold online according to the varying network conditions. Herein, we use the packet loss rate as the QoS metric. In the analytical model, a polynomial fit is employed to deduce the close-form expression of the optimal PCS threshold. In the algorithm design, we construct a balance equation, which root is the optimal PCS threshold. To facilitate the practical implementation, all parameters of the balance equation are expressed with the network conditions that can be measured with the CSMA mechanism. By means of the balance equation, the PCS threshold can be tuned heuristically according to the network conditions and the QoS requirement. One important feature of the proposed algorithm is that it avoids the complicated computation in solving the optimal PCS threshold, and thus it is capable of improving the performance of networks on-line. Extensive simulation results show that the proposed algorithm can make the PCS threshold quickly converge to the value approaching to the theoretical optimal PCS threshold. The unfairness caused by distributed PCS tuning are discussed, and experimental results based on StarEast platform ${ }^{4}$ show that a pure distributed PCS tuning starves the users far from their receivers.

The rest of the paper is organized as follows. In Section II, we present the analytical model to investigate the throughput per user and the packet loss rate. The heuristic algorithm is proposed in Section III. In Section IV, extensive simulation results are given to evaluate the proposed algorithm. A discussion on unfairness is given in Section V. Related work on our topic is summarized in Section VI. Finally, the conclusions are drawn in Section VII.

\footnotetext{
${ }^{3}$ For TCP services, the TCP throughput is considered to be the main QoS metric. It is well known that packet loss degrade the TCP throughput seriously because TCP is based on the principle of "slow-start". Therefore, it is reasonable to measure the QoS by the packet loss rate.

${ }^{4}$ Due to the lack of devices, only a simple scenario with 4 users is considered in this experiment.
}

TABLE I

Fit CoEfFicients ANd Root Mean Square ERror (RMSE)

\begin{tabular}{|c|c|c|c|c|c|}
\hline Data rate & SINR & $a_{1}$ & $a_{2}$ & $a_{3}$ & RMSE \\
\hline $6 \mathrm{Mbps}$ & $6.02 \mathrm{~dB}$ & 0.491 & -1.931 & 1.896 & 0.018 \\
\hline $9 \mathrm{Mbps}$ & $7.78 \mathrm{~dB}$ & 0.476 & -1.969 & 2.039 & 0.016 \\
\hline $12 \mathrm{Mbps}$ & $9.03 \mathrm{~dB}$ & 0.44 & -1.909 & 2.075 & 0.015 \\
\hline $18 \mathrm{Mbps}$ & $10.79 \mathrm{~dB}$ & 0.37 & -1.741 & 2.053 & 0.015 \\
\hline $24 \mathrm{Mbps}$ & $17.04 \mathrm{~dB}$ & 0.169 & -1.118 & 1.839 & 0.029 \\
\hline $36 \mathrm{Mbps}$ & $18.80 \mathrm{~dB}$ & 0.13 & -0.964 & 1.766 & 0.034 \\
\hline $48 \mathrm{Mbps}$ & $24.05 \mathrm{~dB}$ & 0.014 & -0.413 & 1.379 & 0.038 \\
\hline $54 \mathrm{Mbps}$ & $24.56 \mathrm{~dB}$ & 0.002 & -0.349 & 1.325 & 0.036 \\
\hline
\end{tabular}

\section{Theoretical Analysis of The Optimal PCS THRESHOLD}

IEEE 802.11 based wireless networks have two types of modes: infrastructure mode and independent mode (also called ad hoc mode). Due to the limited space, we only concentrate on the ad hoc mode, but the conclusion can be easily extended to the scenario with the infrastructure mode.

\section{A. System Model}

Considering a network with $M$ users, we index each user with $u_{i}(i=1,2, . ., M)$. All users are uniformly deployed over the 2-D plane with the density of $\lambda$, and all users transmit data frames and control frames with the same power. With the typical pathloss model [20], the average signal strength at the receiver is expressed as a function of the distance between the transmitter-receiver pair, i.e.

$$
P_{i, j}=\bar{P}\left(\frac{\bar{d}}{d_{i, j}}\right)^{\alpha}
$$

where $\alpha$ denotes the pathloss coefficient, ranging from 2 (free space) to 4 (indoor), $P_{i, j}$ denotes the signal strength at $u_{j}$ received from $u_{i}, d_{i, j}$ denotes the distance between $u_{i}$ and $u_{j}$, and $\bar{P}$ is the reference receiving signal strength as measured at the reference distance $\bar{d}$ (usually 1 meter). Let $P_{j}$ denote the aggregate signal strength detected by $u_{j}$, which consists of signal (from expected transmitter), interference (from unexpected transmitter(s)) and noise. Then, we have

$$
P_{j}=P_{i, j}+P_{I}+P_{N}
$$

where $P_{I}$ is the cumulative interference from multiple concurrent transmitters, and $P_{N}$ is the noise. The intended signal can be correctly decoded with high probability if the signalto-interference-and-noise ratio (SINR) is always beyond a threshold $\delta$ during the transmission time, i.e.,

$$
\frac{P_{i, j}}{P_{I}+P_{N}}>\delta .
$$

Herein, $\delta$ depends on the employed data rate. The reference values of $\delta$ for different data rates in IEEE 802.11a can be found in Table.I.

Let $P_{r}$ and $P_{\mathrm{pcs}}$ denote the receiver sensitivity threshold and the PCS threshold, respectively. Then, the reception radius $R_{r}$ and the PCS radius $R_{c}$ are given by

$$
R_{r}=\bar{d}\left(\frac{\bar{P}}{P_{r}}\right)^{1 / \alpha} \text { and } R_{c}=\bar{d}\left(\frac{\bar{P}}{P_{\mathrm{pcs}}}\right)^{1 / \alpha},
$$


respectively. To avoid collisions, users need to know whether the expected receivers are idle or not before transmitting. Therefore, the receivers within the reception range should be also within the PCS range, i.e., $R_{r} \leq R_{c}$.

For an interference-limited network, it is reasonable to ignore the noise and treat $P_{i, j} / P_{I} \leq \delta$ as the condition of collisions. The interfering radius of link " $u_{i} \rightarrow u_{j}$ ", denoted by $I_{i, j}$, is defined as the maximum distance at which $u_{j}$ can be disturbed by other transmitters. Then, we have

$$
\frac{\bar{P}\left(\bar{d} / d_{i, j}\right)^{\alpha}}{\bar{P}\left(\bar{d} / I_{i, j}\right)^{\alpha}}=\delta \quad \Rightarrow \quad I_{i, j}=d_{i, j} \delta^{1 / \alpha}
$$

An aggressive PCS allows the existence of hidden terminals, i.e., the PCS range of transmitters does not cover the interfering range of receivers completely. A typical scenario is shown in Fig.1, where the circle with dash line represents the PCS range and the circle with solid line represents the interfering range. The region within the interfering range but outside the PCS range is called hidden region (the shadow region in Fig.1). The users within the hidden region are called the hidden terminals of the link (" $u_{i} \rightarrow u_{j}$ " in Fig.1). The area of the hidden region depends on the distance of the transmitterreceiver pair and the PCS radius, and is expressed as follows [16]:

$$
\begin{aligned}
& A_{H}\left(d_{i, j}, R_{c}\right)= \\
& \left\{\begin{array}{r}
0, \quad R_{c} \geq d_{i, j}\left(\delta^{1 / \alpha}-1\right) ; \\
\frac{b d_{i, j}^{2} \delta^{2 / \alpha}}{2}+d_{i, j} R_{c}\left|\sin \frac{a}{2}\right|-\frac{a R_{c}}{2}, \\
d_{i, j}\left(\delta^{1 / \alpha}-1\right) \leq R_{c}<d_{i, j}\left(\delta^{1 / \alpha}+1\right) ; \\
\pi\left(d_{i, j}^{2} \delta^{2 / \alpha}-R_{c}^{2}\right), \quad R_{r}<R_{c}<d_{i, j}\left(\delta^{1 / \alpha}-1\right) .
\end{array}\right.
\end{aligned}
$$

where

$$
\left\{\begin{array}{l}
a=2 \cos ^{-1}\left(\frac{R_{c}^{2}-d_{i, j}^{2}\left(\delta^{2 / \alpha}-1\right)}{2 d_{i, j} R_{c}}\right), \\
b=2 \pi-2 \cos ^{-1}\left(\frac{d_{i, j}^{2}\left(\delta^{2 / \alpha}+1\right)-R_{c}^{2}}{2 \delta^{1 / \alpha} d_{i, j}^{2}}\right) .
\end{array}\right.
$$

The existence of hidden terminals degrades the reliability of transmissions, which results in the waste of wireless resource due to retransmissions. From (4), the area of the hidden region, which is in proportion to the number of hidden terminals, decreases with $R_{c}$. Therefore, the frame loss rate decreases with $R_{c}$ as well. However, the number of "exposed terminals"5 increases with $R_{c}$, which depresses the spatial reuse efficiency and enforces the considered user to compete with more users for channel access.

Therefore, to optimize the throughput per user, it is important to tune the PCS threshold so as to achieve an efficient tradeoff between hidden terminals and exposed terminals. In the following of this section, we will investigate the relationship between the throughput per user and the PCS threshold. To avoid asymmetric PCS setting, which will result in asymmetric links and decreases the throughput seriously

\footnotetext{
5"Exposed terminal" is the user that is prevented from transmissions due to being in the PCS range, although it in fact would not have disrupted the expected transmissions.
}

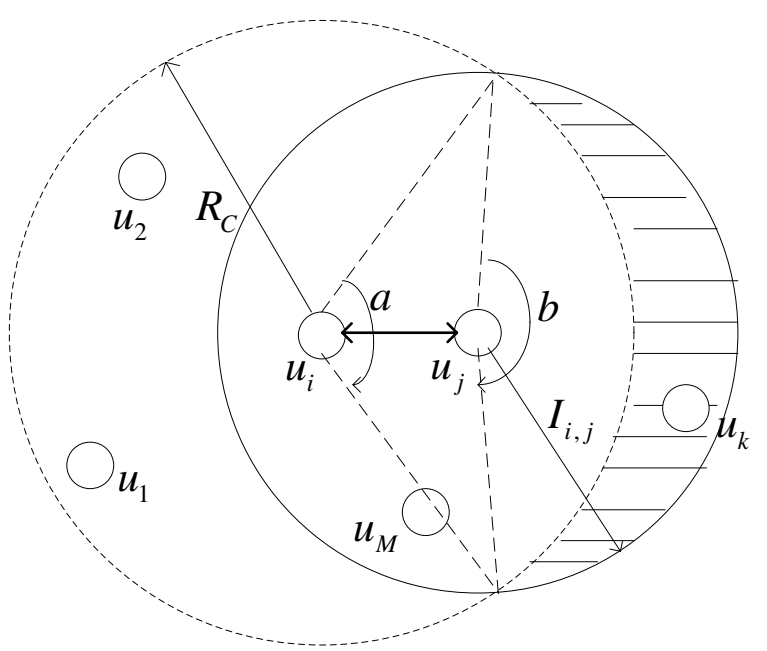

Fig. 1. Sketch for the relationship between carrier sensing range and interfering range (the region enveloped with solid line is interfering range, the region enveloped with dash line is carrier sensing range, and the shadow region is hidden region.)

[19], all users tune the PCS threshold to the same value according to the reception radius and the user density.

\section{B. Throughput per user}

From IEEE 802.11 standard, the backoff time decrement is stopped when the channel is sensed busy, and thus the time interval between two consecutive time slot beginnings may be much longer than the slot time size $\sigma$, as it may include a packet transmission. For the convenience of analysis, we define a virtual time slot which refers to either the (constant) value, and the (variable) time interval between two consecutive backoff time counter decrements.

Let $S$ denote the throughput per user, defined as the average payload transmitted by an arbitrary user in a virtual time slot, i.e.,

$$
S=\frac{\text { E[payload transmitted by the user in a time slot }]}{\text { E[length of a virtual time slot }]},
$$

where $\mathrm{E}[x]$ represents the expected value of $x$. To calculate $S$, let us analyze the behaviors of the user in a time slot. Let $P_{s}$ denote the probability that the user transmits a data frame successfully in a time slot. Let variable $L$ denote the payload size of a data frame. Then, the average payload transmitted successfully by the considered user in a virtual time slot is given by $P_{s} \mathrm{E}[L]$. The channel has three states: busy due to a successful transmission, busy due to a collision, and idle. Let $P_{s}^{*}, P_{c}$, and $P_{i}$ denote the probabilities that the channel stays in each state, respectively. Then, we get the expression of $S$ as follows:

$$
S=\frac{P_{s} \mathrm{E}[L]}{P_{s}^{*} T_{s}+P_{c} T_{c}+P_{i} \sigma},
$$

where $T_{s}$ is the average time of a successful transmission, and $T_{c}$ is the average time of a collision. From [12], we have

$$
\left\{\begin{array}{l}
T_{s}=H+\mathrm{E}[L] / r+T_{A C K}+\mathrm{SIFS}+\mathrm{DIFS} \\
T_{c}=H+\mathrm{E}[L] / r+\mathrm{DIFS}
\end{array}\right.
$$


where $H$ is the transmission time of a frame header (consists of a physical header and a MAC header), $r$ is data rate, and $T_{A C K}$ is the transmitting time of an ACK frame. In addition, SIFS and DIFS are a short interframe space and a distributed interframe space, respectively.

To simplify the analysis, we consider a saturation scenario where each user always has a data frame available for transmission. We use $p$-persistent CSMA to approach to the behaviors of the CSMA/CA mechanism with exponential backoff employed in the IEEE 802.11 standard. Let $p$ denote the probability that a user transmits in a given time slot. Let $P_{L}$ denote the frame loss rate. Then, we obtain the expressions of the parameters in (6) as follows ${ }^{6}$ :

$$
\left\{\begin{array}{l}
P_{s}=p\left(1-P_{L}\right), \\
P_{s}^{*}=n_{c} P_{s}=n_{c} p\left(1-P_{L}\right), \\
P_{i}=(1-p)^{n_{c}} \\
P_{c}=1-n_{c} p\left(1-P_{L}\right)-(1-p)^{n_{c}},
\end{array}\right.
$$

where $n_{c}$ is the average number of users in the PCS range. Because it is assumed that all users are deployed uniformly with density $\lambda$, we have $n_{c}=\left[\lambda \pi R_{c}^{2}\right]$, where $[x]$ represents the nearest integer to $x$. Substituting (8) into (6), we have

$S=\frac{p\left(1-P_{L}\right) \mathrm{E}[L]}{n_{c} p\left(1-P_{L}\right)\left(T_{s}-T_{c}\right)+(1-p)^{n_{c}} \sigma+\left[1-(1-p)^{n_{c}}\right] T_{c}}$

Here, $T_{s}$ is in order of millisecond, and is much larger than $T_{A C K}+$ SIFS (just several decade microsecond). Therefore, from (7) it is reasonable to use $T_{s} \approx T_{c}$ to simplify (6). Then, we have

$$
S=\frac{p \mathrm{E}[L]}{\frac{1}{1-P_{L}}\left\{T_{s}\left[1-(1-p)^{n_{c}}\right]+(1-p)^{n_{c}} \sigma\right\}}
$$

In the expression above, $\mathrm{E}[L], p$, and $T_{s}$ are independent of the PCS threshold, and thus maximizing the throughput per user equals to minimizing the denominator. It is well known that $n_{c}$ is in proportion to the area of the PCS range, and thus the optimization objective is converted to find a $n_{c}$ to minimize the denominator of (10). For the convenience of analysis, we define a function $f\left(n_{c}\right)$ to describe the denominator, i.e.,

$$
f\left(n_{c}\right)=\frac{T_{s}\left[1-(1-p)^{n_{c}}\right]+(1-p)^{n_{c}} \sigma}{1-P_{L}}
$$

From the optimization theory, the function reaches the extremum when $n_{c}$ is the root of $f^{\prime}\left(n_{c}\right)=0$.

From the analysis of [14][16], in the network allowing the existence of hidden terminal, the frame loss rate is dominated by the hidden terminals. In this way, we ignore the frame loss due to simultaneous transmissions in the non-hidden region. From the analysis in our previous work [17], the frame loss rate is determined by the average number of users in the PCS range and that in the hidden region. From (4), the average number of users in the hidden region depends on the PCS radius. We employ a function $P_{L}=g\left(n_{c}\right)$ to describe the

\footnotetext{
${ }^{6}$ There are two reasons that cause a transmission fail: collisions due to the simultaneous transmissions in the interfering range, and collisions due to the concurrent transmissions in the hidden region. The probability of failure due to simultaneous transmissions is given by $1-(1-p)^{n_{c}-1}$, and thus we have $0<P_{s}^{*}<n_{c} p(1-p)^{n_{c}-1}<1$.
}

relation between $P_{L}$ and $n_{c}$. Substituting $P_{L}=g\left(n_{c}\right)$ into $f\left(n_{c}\right)$ and making differentiation with respect to $n_{c}$, we have

$$
\begin{aligned}
f^{\prime}\left(n_{c}\right) & =\frac{\left(\sigma-T_{s}\right)(1-p)^{n_{c}} \ln (1-p)}{1-g\left(n_{c}\right)} \\
& +\frac{g^{\prime}\left(n_{c}\right)\left\{T_{s}\left[1-(1-p)^{n_{c}}\right]+(1-p)^{n_{c}} \sigma\right\}}{\left(1-g\left(n_{c}\right)\right)^{2}}
\end{aligned}
$$

The Taylor progression of $\ln (1-p)$ is $\sum_{k=1}^{\infty} \frac{(-p)^{k}}{k}$. Due to $p \ll 1$, we use $-p+\frac{p^{2}}{2}$ to approximate the value of $\ln (1-p)$. In addition, we use $n_{c} p(1-p)^{n_{c}-1}+\frac{n_{c}\left(n_{c}-1\right)}{2} p^{2}(1-p)^{n_{c}-2}$ to approximate to the value of $1-(1-p)^{n_{c}}$. Because $g\left(n_{c}\right)$ is always smaller than 1 , from $f^{\prime}\left(n_{c}\right)=0$ we have

$$
\begin{aligned}
& \left(1-g\left(n_{c}\right)\right)\left(1-\frac{p}{2}\right)\left(T_{s}-\sigma\right) \\
& +g^{\prime}\left(n_{c}\right)\left[\frac{n_{c}}{1-p} T_{s}+\frac{n_{c}\left(n_{c}-1\right) p}{2(1-p)^{2}} T_{s}+\frac{\sigma}{p}\right]=0
\end{aligned}
$$

The condition of a successful transmission occurs is that there are no concurrent transmissions in the hidden region, i.e., all users in the hidden region keep idle in both the last data frame transmission interval and the current interval. From the principle of CSMA, if a user starts a transmission, all users within the related PCS range will not start new transmissions. Therefore, we have

$$
g\left(n_{c}\right)=\frac{h\left(n_{c}\right)}{n_{c}}
$$

where $h\left(n_{c}\right)$ is the average number of users in the hidden region which depends on $n_{c}$. For the considered user, the potential receivers are uniformly distributed within the circle centered as the considered user, the maximum distance of transmitter-receiver is $R_{r}$. The probability density function of the transmitter-receiver distance is given by $q(r)=\frac{2 r}{R_{r}^{2}}$. Then, $g\left(n_{c}\right)$ is given by

$$
g\left(n_{c}\right)=\frac{1}{\pi R_{c}^{2}} \int_{0}^{R_{r}} q(r) A_{H}\left(r, R_{c}\right) \mathrm{d} r .
$$

Substituting (4) into (15), we get that $g\left(n_{c}\right)$ arbitrarily depends on $\frac{R_{c}}{R_{r}}$. By means of numerical solutions, for fixed pathloss coefficient $\alpha=4$ (indoor environment) we further derive the relation between $g\left(n_{c}\right)$ and $\frac{R_{c}}{R_{r}}$ for various data rates. Herein, different data rates correspond to different SINR requirements for successful decoding. We approach to the numerical results with a quadratic polynomial. Consequently, the value of $g\left(n_{c}\right)$ is approximately given by $g\left(n_{c}\right)=a_{1}\left(\frac{R_{c}}{R_{r}}\right)^{2}+a_{2} \frac{R_{c}}{R_{r}}+a_{3}$. The values of $\left\{a_{1}, a_{2}, a_{3}\right\}$ for various data rates are listed in Table.I. To clarify the fit effect, the root mean square errors are also listed. From Table.I and Fig.2, it is observed that the relation between $g\left(n_{c}\right)$ and $\frac{R_{c}}{R_{r}}$ can be approached properly with the quadratic polynomial.

Then, we derive $g^{\prime}\left(n_{c}\right)$ by differentiation directly. Substituting $g\left(n_{c}\right)$ and $g^{\prime}\left(n_{c}\right)$ into (12), we can solve the optimal $n_{c}$ corresponding to the maximum throughput from $f^{\prime}\left(n_{c}\right)=0$, which is in fact a polynomial solution. Let $n_{c}^{*}$ denote the root of $f^{\prime}\left(n_{c}\right)=0$. Then, the optimal carrier sensing radius is 


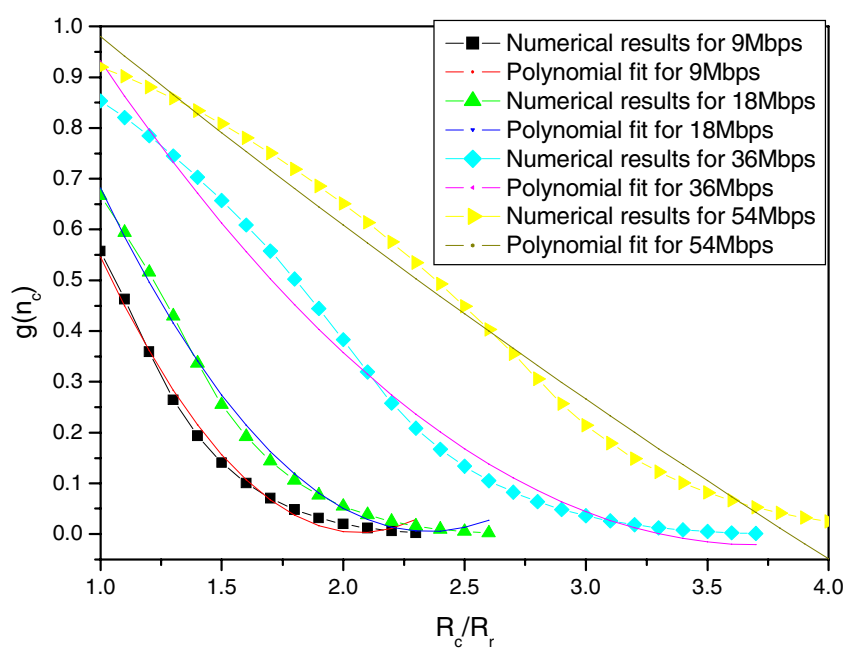

Fig. 2. $g\left(n_{c}\right)$ vs. $R_{c} / R_{r}$ :numerical results and polynomial fit.

$R_{c}=\sqrt{n_{c}^{*} / \lambda \pi}$, and consequently the optimal PCS threshold is given by

$$
P_{\mathrm{pcs}}=\bar{P}\left(\bar{d} \sqrt{\frac{\lambda \pi}{n_{c}^{*}}}\right)^{\alpha}
$$

Substituting (16) into (10), we can obtain the throughput per user with the optimal PCS threshold.

\section{QoS Requirement: Packet Loss Rate}

From the viewpoint of application layer, the QoS, which generally consists of delay and packet loss rate, is the main metric of the system. The aggressive PCS increases the throughput which is reversely in proportion to the delay, while it results in high packet loss rate. Therefore, we focus on the packet loss rate.

As mentioned above, the aggressive PCS brings too many MAC-layer retransmissions due to frame loss. From IEEE 802.11 standard, a data packet will be dropped after $m(m=$ 4 or 7 ) retransmissions, which triggers a high-layer packet loss. Therefore, the packet loss rate, which is denoted by $\theta$, is given by $\theta=P_{L}^{m}$. With the parameters described in Section IV, we plot Fig.3 to show the packet loss rate of a link with the distance $R_{r}$. With $\theta=1 \%$, we get that the PCS threshold should be tuned to make $R_{c} / R_{r}>1.3$ for 9 Mbps, $R_{c} / R_{r}>1.5$ for $18 \mathrm{Mbps}, R_{c} / R_{r}>2.3$ for $36 \mathrm{Mbps}$, and $R_{c} / R_{r}>2.8$ for 54 Mbps. With the same parameters, Fig.4 shows the throughput variation. From the figure, it is observed that for $36 \mathrm{Mbps}$ and $54 \mathrm{Mbps}$ the packet loss rate is larger than $\theta$ when the throughput reaches to the maximum. Therefore, in the algorithm design, the effect of packet loss rate should be considered to guarantee the QoS.

\section{QoS-aWare Adaptive Physical Carrier Sensing}

In the previous section, we deducted the optimal PCS threshold corresponding to the maximum throughput per user by means of an analytical model. However, the theoretical results cannot be applied into the practical networks directly due to two reasons: first, there is no centralized point to collect network status information; second, due to the randomness of

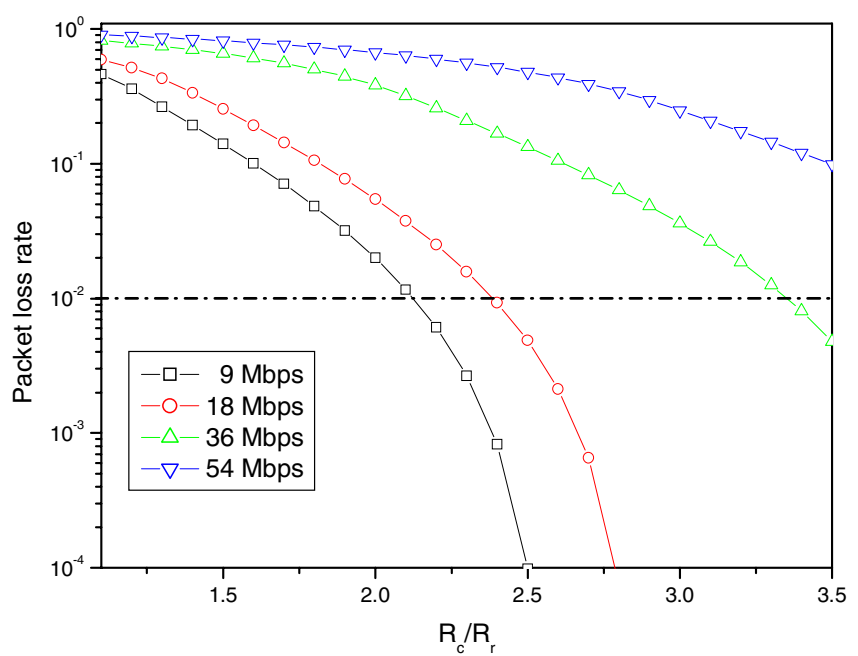

Fig. 3. Packet loss rate for various data rate.

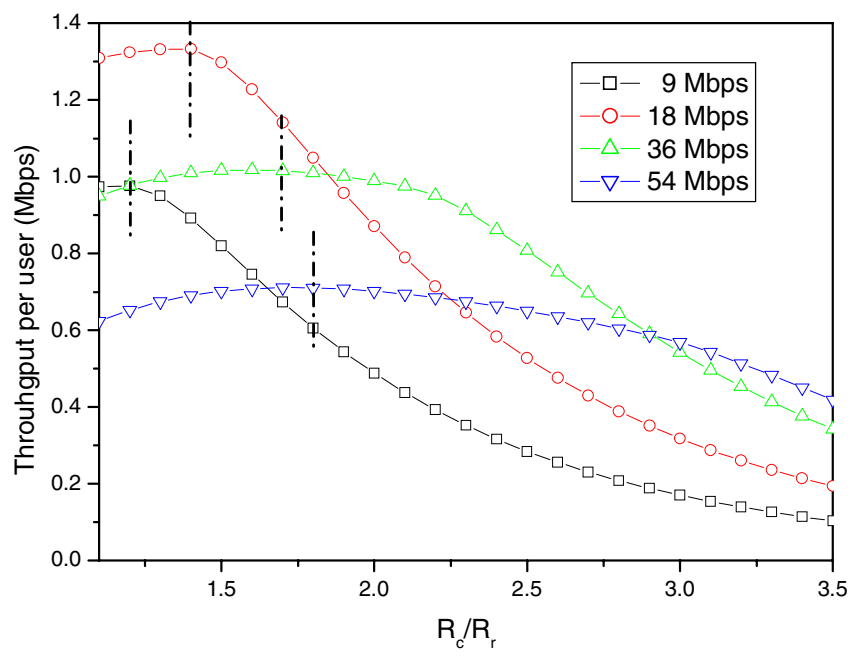

Fig. 4. Throughput per user for various data rate.

the CSMA backoff mechanism, it is very difficult to precisely estimate the network conditions on-line (such as the user density and the transmission probability $p$ ), which are needed to calculate the optimal PCS threshold. Therefore, in this section we further simplify the analytical model, and develop a heuristic algorithm, in which we employ limited information obtained by means of the CSMA mechanism to estimate the network conditions, to adapt the PCS threshold. The QoS requirement is taken into account by bounding the packet loss rate.

By the CSMA mechanism, each user can sense all transmissions occur in its PCS range. In this way, without the help of centralized point each user can maintain the following statistical information on-line:

- $T_{\text {success }}$, the time that the channel is occupied by the successful transmissions of the user itself.

- $T_{\text {capture }}$, the time that the channel is captured due to the transmissions of users within the reception range, which consists of the successful transmissions and the collided transmissions.

- $T_{\text {busy }}$, the time that the channel is busy due to the transmissions of the users within the PCS range, which 
includes the transmissions in the reception range.

- $T_{\text {idle, }}$, the time that the channel is idle due to no transmissions in the PCS range.

- $n_{r}$, the number of users in the reception range.

The purpose of this section is to design a heuristic adaptive PCS threshold tuning algorithm, in which the parameters required to adapt the PCS threshold can be estimated from the information listed above. To meet this target, we should first modify the model developed in the previous section.

Recall expression (12), we employ $-p$ to approximate the value of $\ln (1-p)$, and then we have

$$
\begin{aligned}
f^{\prime}\left(n_{c}\right) & =\frac{\left(T_{s}-\sigma\right) p(1-p)^{n_{c}}}{1-g\left(n_{c}\right)} \\
& +\frac{g^{\prime}\left(n_{c}\right)\left\{T_{s}\left[1-(1-p)^{n_{c}}\right]+(1-p)^{n_{c}} \sigma\right\}}{\left(1-g\left(n_{c}\right)\right)^{2}} .
\end{aligned}
$$

Because $1-g\left(n_{c}\right)$ is always larger than 0 , the root of $f^{\prime}\left(n_{c}\right)=$ 0 can also be solved from

$$
\begin{aligned}
& g^{\prime}\left(n_{c}\right)\left\{T_{s}\left[1-(1-p)^{n_{c}}\right]+(1-p)^{n_{c}} \sigma\right\} \\
& +\left[1-g\left(n_{c}\right)\right]\left(T_{s}-\sigma\right) p(1-p)^{n_{c}}=0 .
\end{aligned}
$$

Dividing both sides of the equation by $T_{s}\left[1-(1-p)^{n_{c}}\right]+$ $(1-p)^{n_{c}} \sigma$, we get that

$$
\frac{\left[1-g\left(n_{c}\right)\right]\left(T_{s}-\sigma\right) p(1-p)^{n_{c}}}{T_{s}\left[1-(1-p)^{n_{c}}\right]+(1-p)^{n_{c}} \sigma}+g^{\prime}\left(n_{c}\right)=0 .
$$

From (10), the first item of (19) can be rewritten as

$$
\frac{\left[1-g\left(n_{c}\right)\right]\left(T_{s}-\sigma\right) p}{T_{s}\left[1-(1-p)^{n_{c}}\right]+(1-p)^{n_{c}} \sigma}=\frac{\left(T_{s}-\sigma\right) T_{\text {success }}}{T_{s}} .
$$

In addition, from (8) we have

$$
\left\{\begin{array}{c}
T_{\text {idle }}=\frac{(1-p)^{n_{c}} \sigma}{(1-p)^{n_{c}} \sigma+\left[1-(1-p)^{n_{c}}\right] T_{s}}, \\
T_{\text {busy }}=\frac{\left[1-(1-p)^{n_{c}}\right] T_{s}}{(1-p)^{n_{c}} \sigma+\left[1-(1-p)^{n_{c}}\right] T_{s}} .
\end{array}\right.
$$

In this way, $(1-p)^{n_{c}}$ can be estimated by

$$
(1-p)^{n_{c}}=\frac{\frac{T_{\text {idle }}}{\sigma}}{\frac{T_{\text {busy }}}{T_{s}}+\frac{T_{\text {idle }}}{\sigma}} .
$$

Then, substituting (20) and (22) into (19), we have

$$
\frac{\left(T_{s}-\sigma\right) T_{\text {success }} T_{\text {idle }}}{\sigma T_{\text {busy }}+T_{s} T_{\text {idle }}}+g^{\prime}\left(n_{c}\right)=0 .
$$

Because it is very difficult to identify the closed-form expression of $g\left(n_{c}\right)$, the precise value of optimal $n_{c}$ cannot be computed directly from the equation above. In this paper, a heuristic adaptive tuning on the PCS threshold is considered. As defined above, $g\left(n_{c}\right)$ is the ratio of the number of collided packets and the total number of packets transmitted. In the reception range, the average number of data frames transmitted in a given time interval is in proportion to the sensed $T_{\text {capture }}$, and the average number of successful transmissions of a single user is in proportion to $T_{\text {success. }}$. The number of users in the reception range is known as $n_{r}$ in advance. In this way, we can estimate $g\left(n_{c}\right)$ by

$$
g\left(n_{c}\right)=\frac{T_{\text {capture }}-n_{r} T_{\text {success }}}{T_{\text {capture }}} .
$$

In addition, the value of $n_{c}$ can be estimated by

$$
n_{c}=n_{r} \frac{T_{\text {busy }}}{T_{\text {capture }}}
$$

To track the changes in network status on-line, our algorithm updates the estimates of the network status at intervals. In the simulation, the interval is set as $100 \mathrm{~ms}$. During the $i$ th interval, the values of $\left\{T_{\text {success }}, T_{\text {capture }}, T_{\text {busy }}, T_{\text {idle }}\right\}$ are estimated by statistical measurement and denoted by $\left\{T_{\text {success }}^{(i)}, T_{\text {capture }}^{(i)}, T_{\text {busy }}^{(i)}, T_{\text {idle }}^{(i)}\right\}$. Then, $\left\{T_{\text {success }}, T_{\text {capture }}, T_{\text {busy }}, T_{\text {idle }}\right\}$ are updated after the $i$ th interval as follows

$$
\left\{\begin{array}{rl}
T_{\text {success }} & =\gamma T_{\text {success }}+(1-\gamma) T_{\text {success }}^{(i)} \\
T_{\text {capture }} & =\gamma T_{\text {capture }}+(1-\gamma) T_{\text {capture }}^{(i)} \\
T_{\text {busy }} & =\gamma T_{\text {busy }}+(1-\gamma) T_{\text {busy }}^{(i)} \\
T_{\text {idle }} & =\gamma T_{\text {idle }}+(1-\gamma) T_{\text {idle }}^{(i)}
\end{array},\right.
$$

where $\gamma$ is a smooth factor, which is widely adopted in network protocols to obtain reliable estimates. Extensive simulations show that $\gamma=0.9$ is a good compromise between accuracy and promptness. After obtaining $\left\{T_{\text {success }}, T_{\text {capture }}, T_{\text {busy }}, T_{\text {idle }}\right\}$, the frame loss rate $g^{(i)}\left(n_{c}\right)$ and $n_{c}^{(i)}$ can be updated by (24) and (25). Then, the PCS threshold can be dynamically tuned according to the value of (23). The pseudo code of the proposed algorithm is shown below. Herein, $\eta$ is a constant used as learning-rate coefficient which determines the convergence speed and the stability of the algorithm, and $P_{L}^{*}$ represents the packet loss rate estimated by the same method as (26). The key step is updating the PCS threshold in Step 9. Here, the expression $\frac{\left(T_{s}-\sigma\right) T_{\text {success }} T_{\text {idle }}}{\sigma T_{\text {busy }}+T_{s} T_{\text {idle }}}+$ $g^{\prime}\left(n_{c}\right)$, which comes from (23), indicates the tuning direction, because the PCS threshold is beyond the optimal PCS computed from (23) when $\frac{\left(T_{s}-\sigma\right) T_{\text {success }} T_{\text {idle }}}{\sigma T_{\text {busy }}+T_{s} T_{\text {idle }}}+g^{\prime}\left(n_{c}\right)<0$ and vice vase. In addition, to bound the frame loss rate, a constraint of $\left(\theta-P_{L}^{*}\right)$ is included in updating the PCS threshold.

Heuristic Algorithm
1. Initialize $P_{\mathrm{pcs}}, n_{r}$
2. Do
3. Obtain the statistics of $\left\{T_{\text {success }}^{(i)}, T_{\text {capture }}^{(i)}, T_{\text {busy }}^{(i)}, T_{\text {idle }}^{(i)}\right\}$
4. Update $\left\{T_{\text {success }}, T_{\text {capture }}, T_{\text {busy }}, T_{\text {idle }}\right\}$ by $(26)$
5. Estimate $g^{(i)}\left(n_{c}\right)$ by $(24)$
6. Estimate $n_{c}^{(i)}$ by $(25)$
7. Calculate $g^{\prime}\left(n_{c}\right)=\frac{g^{(i)}\left(n_{c}\right)-g^{(i-1)}\left(n_{c}\right)}{n_{c}^{(i)}-n_{c}^{(i-1)}}$
8. Update the the frame loss rate $P_{L}^{*}$
9. Update the carrier sensing threshold by
10. End do

\section{Simulation RESUlts}

In this section, we take IEEE 802.11a protocol for example to evaluate the performance of the proposed heuristic algorithm with NS-2 simulator [21]. The parameters of the physical layer and MAC layer used in the simulation are listed in Table.II. The reception radius is normalized as $R_{r}=1$ unit length, and all users are deployed uniformly with user density 
TABLE II

PARAMETERS FOR WLANS

\begin{tabular}{|c|c|}
\hline Parameter & value \\
\hline transmission time for Physical header(us) & 8 \\
MAC header (bytes) & 28 \\
SIFS time (us) & 16 \\
Slot time (us) & 9 \\
DIFS time (us) & 34 \\
\hline CW $_{\min }$ & 31 \\
$\mathrm{CW}_{\max }$ & 127 \\
\hline$\eta$ & 2 \\
\hline Packet length(bytes) & 1024 \\
\hline
\end{tabular}

$\lambda$. For each simulation scenario, the simulation time is 300 seconds, and the results are obtained via averaging values from 10 different runs with different seeds. The physical layer data rates used in the simulation follow the specifications of IEEE 802.11a protocol, and the SINR required for each data rate is the same to that listed in Table.I.

In the rest part of this section, we firstly explore the convergence of the proposed heuristic algorithm. After that, we implement extensive simulations to compare the performance of the proposed heuristic algorithm with the traditional PCS threshold setting and the optimal PCS threshold setting.

\section{A. Convergence of Heuristic Algorithm}

First of all, we investigate the convergence of the proposed algorithm. For a given pair of transmitter-receiver, we plot the throughput variation with respect to time. For the purpose of comparison, the throughput calculated with the optimal PCS threshold, which is solved with the theoretical method, is indicated as well. As shown in Fig.5, the convergence speed increases with the learning-rate coefficient $\eta$ : for $\eta=1$, the user costs $9 \mathrm{~s}$ to reach to the stable state; for $\eta=4$, the user costs $2.5 \mathrm{~s}$ to reach to the stable state. On the other hand, the oscillation extent increases with $\eta$ as well, and moreover the average throughput degrades with $\eta$ in the stable state. This is mainly because that a larger learning-rate coefficient results in a larger oscillation of the PCS threshold around the optimal one, which degrades the probability of attaining the optimum. From the consideration of the joint effect of the convergence speed and the oscillation extent, we employ $\eta=2$ as the default value in our simulations.

In addition, we note that an obvious gap (nearly 10\%) between the average throughput obtained with our algorithm and that calculated with the optimal PCS threshold. The gap mainly results from two reasons: one is that the simulation is based on an exponential backoff mechanism in which the transmission probability is not independent of the frame loss rate, and thus there is a small error in estimating the optimal PCS threshold; the other is that the frame loss due to simultaneous transmissions is ignored in calculating the throughput with the optimal PCS, but in the simulation it is considered.

\section{B. Performance Comparison}

In this section, we implement extensive simulations to investigate the performance of the proposed algorithm compared

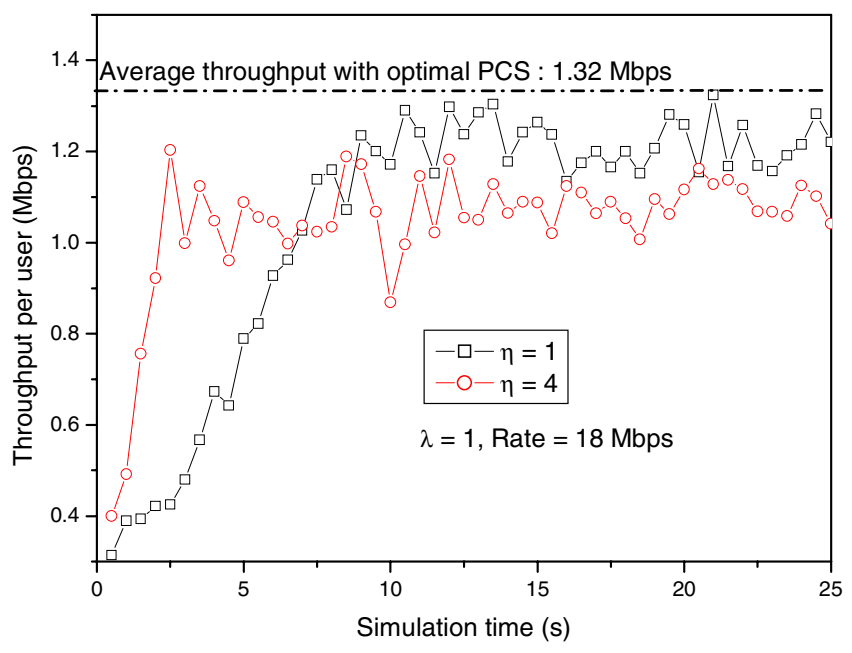

Fig. 5. Stability of the heuristic algorithm.

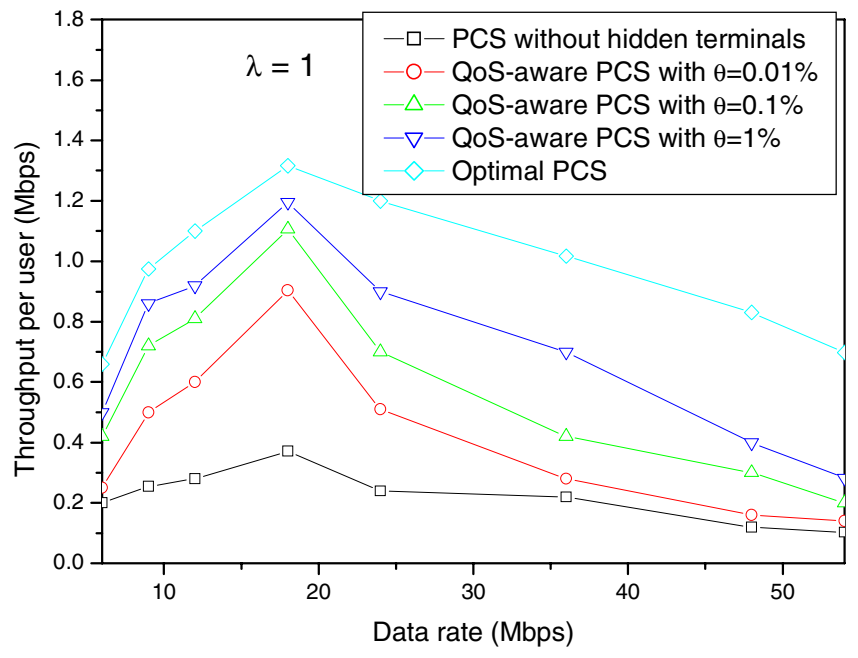

Fig. 6. Performance comparison for various data rate.

to the PCS setting without hidden terminals and optimal PCS setting. To keep the fairness of comparison, we consider the throughput of a fixed pair of "transmitter-receiver" with the distance being $R_{r}$. Herein, the PCS setting without hidden terminals refers to that configuring the PCS threshold to make the PCS range exactly cover the interfering range, and the optimal PCS setting refers to that corresponds to the maximum throughput ignoring the QoS requirement, which is obtained by exhaustive searching.

We first investigate the impact of data rate on the throughput. As shown in Fig.6, the proposed algorithm outperforms the PCS setting without hidden terminals for various data rates, and the performance gap compared to the optimal PCS decreases with the QoS requirement in terms of packet loss rate. A surprising result shown in Fig.6 is that the data rate of $18 \mathrm{Mbps}$ achieves the maximum throughput, which approves that the throughput per user in a large-scale network is not determined by the data rate solely. Due to the limited space, only the performance of a typical data rate $18 \mathrm{Mbps}$ is employed in the following simulations.

Fig.7 shows the throughput per user versus the user density. It is observed that the proposed heuristic algorithm outper- 


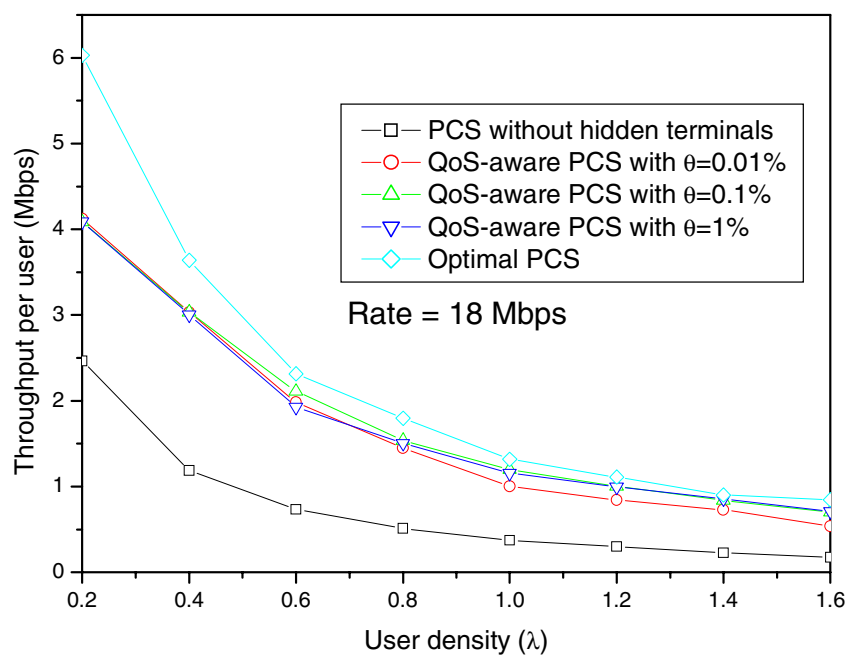

Fig. 7. Performance comparison for various user density.

forms the PCS setting without hidden terminals significantly. In particular, in the scenario with higher user density, the proposed algorithm with various QoS requirement is capable of approaching to the optimal PCS properly because the packet loss corresponding to the optimal PCS is not beyond the QoS requirement in these scenarios. Differently, in the scenario with lower user density, the performance of the proposed algorithm varies with the QoS requirement, and for the same user density the throughput per user increases with $\theta$. In the scenario with lower user density, the packet loss rate corresponding to the optimal PCS is beyond the QoS requirement $(\theta)$, and thus the PCS tuned by the proposed algorithm with larger $\theta$ can approach to the optimal one better than that tuned with smaller $\theta$.

\section{FAIRNESS DisCUSSION}

Instead of employing a pure distributed tuning algorithm, in which each user tune the PCS threshold independently according to the distance of "transmitter-receiver", our algorithm makes all users set the same PCS threshold according to the reception radius and the user density. Such a scheme has two important features: one is that it enables each user has the same transmission probability; the other is that it can bound the frame loss rate. Taking the scenario employed in Section IV for example, we plot the throughput of the users with different "transmitter-receiver" distances in Fig.8. Herein, $d_{i, j}$ represents the distance of the "transmitter-receiver" pair. From the figure, it is observed that with the same PCS threshold the users with smaller $d_{i, j}$ can obtain higher throughput. This is mainly because the frame loss rate decreases with $d_{i, j}$, and the worst case is that $d_{i, j}$ is equal to the reception radius. Moreover, when $d_{i, j}$ shrinks to the value that makes the PCS range cover the corresponding interference range completely, i.e., no hidden terminals, the throughput maintains in a stable level. Although the proposed algorithm is not an absolute throughput fairness scheme, the degree of unfairness is controllable.

Alternatively, if a pure distributed PCS tuning algorithm is employed, the unfairness issue will make the whole network

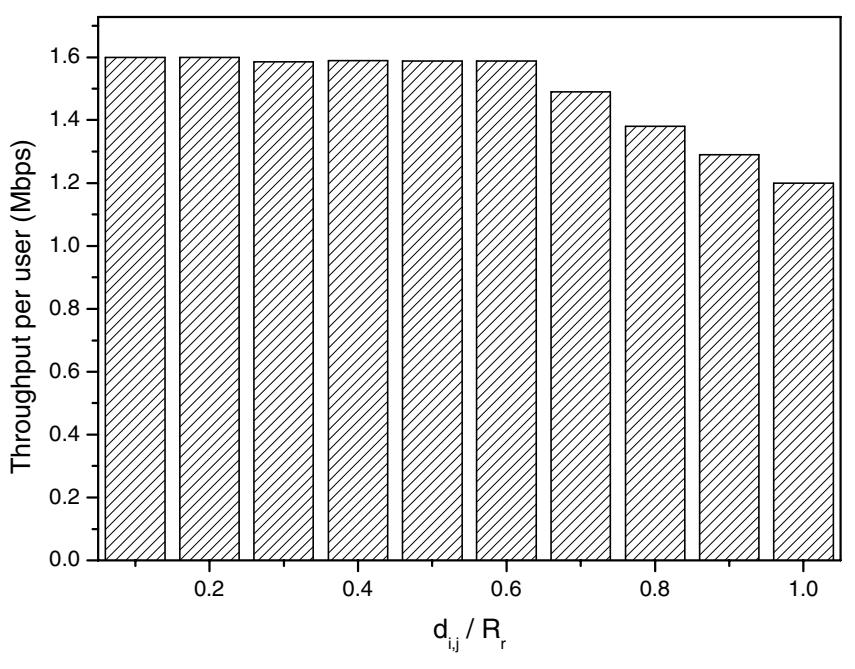

Fig. 8. Throughput comparison for the users with different distance to the receivers.

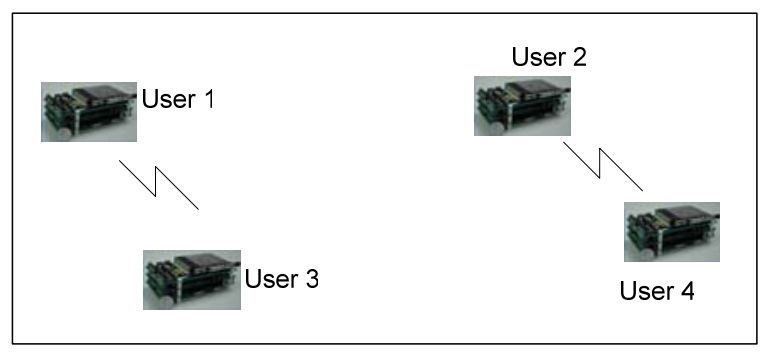

Fig. 9. Topology of testbed measurement.

vulnerable. $^{7}$ The user with a smaller "transmitter-receiver" distance will employ a larger PCS threshold, and can get higher throughput than others due to less users within the PCS range. However, the user with a larger "transmitter-receiver" distance will employ a smaller PCS threshold, and they are vulnerable due to being out of the PCS range of those with larger PCS threshold. Therefore, the throughput unfairness is more serious under pure distributed PCS tuning algorithm. Moreover, the degree of unfairness is topology related and difficult to be estimated.

For this issue, we make a practical experiment with a testbed based on Intel StarEast platform. The environment is FIT building of Tsinghua University, and the topology is shown as Fig.9. We concentrate on the performance of two "transmitter-receiver" pairs ${ }^{8}$, indexed as $\left(u_{1}, u_{3}\right)$ and $\left(u_{2}, u_{4}\right)$. Herein, the channel condition from $u_{1}$ to $u_{3}$ and that from $u_{2}$ to $u_{4}$ are measured by the received signal strength indication $R S S I_{1}(\mathrm{dBm})$ and $R S S I_{2}(\mathrm{dBm})$, which are provided by physical interfaces. From (1), $R S S I_{1}$ and $R S S I_{2}$ indicate the distance from $u_{1}$ to $u_{3}$ and that from $u_{2}$ to $u_{4}$, respectively. With a pure distributed PCS algorithm, $u_{1}$ and $u_{2}$ tune their PCS threshold independently following the proposed algorithm. The experimental results for various $R S S I_{1} / R S S I_{2}$ are shown in Fig.10. It is observed that

\footnotetext{
${ }^{7}$ Similar resource management schemes, such as pure distributed rate and power control schemes, bring same problem as well.

${ }^{8}$ In the building, there already exist many WLAN devices working in the same channel with our testbed, which traffics are considered to be the background traffic of our testbed.
} 


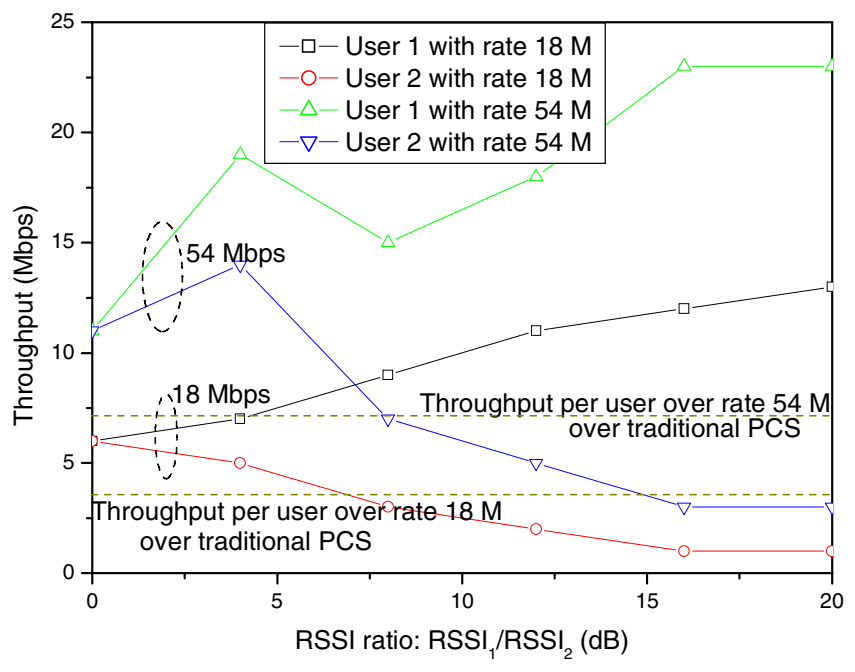

Fig. 10. Experimental results for the pure distributed PCS tuning.

the throughput gap between $u_{1}$ and $u_{2}$ increases with the $R S S I_{1} / R S S I_{2}$ which indicates the degree of asymmetry between $\left(u_{1}, u_{3}\right)$ and $\left(u_{2}, u_{4}\right)$. In particular, for both 18Mbps and $54 M b p s, u_{1}$ and $u_{2}$ get the same throughput when $R S S I_{1}=R S S I_{2}$. Under this condition, the pure distributed PCS in fact behaves the same as our algorithm, and the obtained throughput is much larger than that with traditional PCS (without hidden terminals) indicated with dash line. Differently, $u_{2}$ is nearly starved when $R S S I_{1} / R S S I_{2}$ increases up to $15 \mathrm{~dB}$. Moreover, we find that the throughput sum of $u_{1}$ and $u_{2}$ with $R S S I_{1} / R S S I_{2}>15 d B$ is smaller than that with $R S S I_{1} / R S S I_{2}=4 d B$. Therefore, the pure distributed PCS tuning results in serious unfairness, which starves some users and degrade the total throughput. Similar issues have been investigated in [19].

For the fairness issue, a potential solution is to tune down the transmit power of the user with shorter "transmitterreceiver" distance, and then the interference to neighbor users with longer distances can be depressed. Therefore, a joint control of the PCS threshold and the transmit power should be considered. It is out of the scope of this paper, and will be investigated in the future work.

\section{RELATED WORK}

In the past decade, much attention has been attracted to improve the efficiency of the MAC protocol. For IEEE 802.11 based wireless networks, early work concentrates on optimizing the parameters of MAC protocol, such as transmit power [6][7], RTS/CTS handshaking [8], frame size, contention windows [9][10], and data rate [11], to improve the throughput. However, with the increasingly development of network scale, PCS, which determines the spatial reuse efficiency directly, has been an important research topic.

Existing works mainly concentrate on conservative PCS, which is designed to eliminate the hidden terminal problem completely, i.e., the PCS range covers the whole interfering range. With the conservative PCS threshold the frame loss rate is very low due to no hidden terminals, and then the throughput per user is mainly dependent of the spatial reuse efficiency. With extensive simulation results, [1] first showed that the spatial reuse efficiency could be significantly improved by tuning the PCS threshold. Later on, to maximize the spatial reuse efficiency, [2][4] proposed to tune the PCS threshold to make the PCS range exactly cover the interfering range. In [3], the author developed an analytical model and simulation platform to study the relationship among the MAC overhead, data rate, and PCS threshold. Their research showed that the MAC overhead plays an important role on the choice of optimal PCS range. If MAC overhead is not taken into account properly in determining the optimal PCS range, the throughput can suffer a significant loss. In addition, the effect of PCS on multi-rate and multi-hop wireless ad hoc networks was discussed in [15]. However, so far less attention has been paid on aggressive PCS.

Among the few, in [16], by means of a Markov model and extensive simulations the authors investigated the effect of an aggressive PCS threshold on the frame loss rate and the aggregate one-hop throughput. The results showed that the aggressive PCS threshold could obtain higher throughput per user compared to that with conservative PCS threshold. However, due to the essential deficiency of the Markov model, the relationship between the PCS threshold and the throughput was just expressed with a set of equations but no closedform solutions. The method for calculating the optimal PCS threshold was not addressed in [16], and no PCS on-line tuning algorithm was proposed, which we believe are the main contributions of this paper.

In [14], we developed an experimental testbed based on Intel PRO/Wireless 2200 MiniPCI card to investigate the impact of aggressive PCS threshold on the throughput per user. Based on the testbed, we proposed a combination of Receiving-Sensitivity adaptation to reduce strong-last collision and a PCS adaptation to balance the hidden/exposed terminal problems. We demonstrated through testbed measurement that frame loss rate is an effectively available metric on today 802.11 hardware for the PCS adaption. Due to no analysis on the optimization of the PCS threshold, the proposed method cannot set the PCS threshold to the optimum but just tune it to make the frame loss rate fall into a region given in advance.

\section{CONCLUSION}

The PCS threshold is the key of the tradeoff between the hidden terminal problem and the exposed terminal problem, and thus optimizing the PCS threshold is an important and promising approach for enhancing the throughput per user. In this paper, we developed an analytical model to investigate the impact of the aggressive PCS threshold on the throughput per user. With a few reasonable simplification and a polynomial fit, we obtained a closed-form expression of the optimal PCS threshold. Moreover, based on the model, the access delay was studied as well. Then, according to the characteristics of practical networks, we proposed a heuristic algorithm, in which the parameters required to computing the optimal PCS threshold are estimated with the limited information obtained by the CSMA mechanism, to tune the PCS threshold dynamically to approach to the optimum. Extensive simulation results are given to validate the feasibility and efficiency of our algorithm. 


\section{ACKNOWLEDGMENT}

This research was supported in part by Intel corporation, the NSFC oversea Young Investigator grant under grant No. 60629203 , the National 863 Program of China under Grant No. 2006AA01Z228, the Key Project of Guangzhou Municipal Government Guangdong/Hong Kong Critical Technology grant 2006Z1-D6131, and the HKUST RPC06/07.EG05.

\section{REFERENCES}

[1] X. Guo, S. Roy, and W. S. Conner, "Spatial reuse in wireless ad-hoc networks," in Proc. VTC2003-Fall, vol. 3, pp. 1437-1442, Oct. 2003.

[2] J. Zhu, X. Guo, L. L. Yang, and W. S. Conner, "Leveraging spatial reuse in 802.11 mesh networks with enhanced physical carrier sensing," in Proc. ICC' 04, June 2004.

[3] X. Yang and N. H. Vaidya, "On physical carrier sensing in wireless ad hoc networks," in Proc. IEEE INFOCOM 2005, vol. 4, pp. 2525-2535, Mar. 2005.

[4] J. Zhu, et al., "Adaptive physical carrier sensing to maximize spatial reuse in 802.11 mesh networks," Wiley Wireless Communications and Mobile Computing, vol. 4, pp. 933-946, 2004.

[5] A. Akella, G. Judd, S. Seshan, and P. Steenkiste, "Self-management in chaotic wireless deployments," in Proc. MobiCom'05, Aug. 2005.

[6] J. Chen, S.H. Chan, Q. Zhang, W. Zhu, and J. Chen, "PASA: power adaptation for starvation avoidance to deliver wireless multimedia," IEEE J. Sel. Areas Commun., vol. 21, no. 10, pp. 1663-1673, Dec. 2003.

[7] A. Muqattash and M. Krunz, "POWMAC: a single-channel powercontrol protocol for throughput enhancement in wireless ad hoc networks," IEEE J. Select. Areas Commun., vol. 23, no. 5, May 2005.

[8] F. Ye and B. Sikdar, "Distance-aware virtual carrier sensing for improved spatial reuse in wireless networks," in Proc. IEEE GLOBECOM, pp. 3793-3797, Dallas, TX, Nov. 2004.

[9] F. Cali, M. Conti, and E. Gregori, "Dynamic tuning of the IEEE 802.11 protocol to achieve a theoretical throughput limit," IEEE/ACM Trans. Networking, vol. 8, no. 6, pp. 785-799, Dec. 2000.

[10] F. Cali, M. Conti, and E. Gregori, "IEEE 802.11 protocol: design and performance evaluation of an adaptive backoff mechanism," IEEE $J$. Select. Areas Commun., vol. 18, no. 9, pp. 1774-1786, Sept. 2000.

[11] Y. Zhu and Z. Niu, "User-aware rate adaptive control for IEEE 802.11based ad hoc networks," in Proc. Globemcom 2005, St. Louis, MS, vol. 5, pp. 2605-2609, Nov. 2005.

[12] IEEE 802.11, Part 11: Wireless LAN Medium Access Control (MAC) and Physical Layer (PHY) specifications, IEEE standard for information technology telecommunications and information exchange between systems-local and metropolitan area networks-specific requirements, 1999.

[13] K. Whitehouse, A. Woo, F. Jiang, J. Polastre, and D. Culler, "Exploiting the capture effect for collision detection and recovery," in Proc. Second IEEE Workshop on Embedded Networks Sensors (EmNetS-II), Sydney, Australia. May 2005.

[14] J. Zhu, B. Metzler, X. Guo, and Y. Liu, "Adaptive CSMA for scalable network capacity in high-density WLAN: a hardware prototyping approach," in Proc. INFOCOM'06, Barcelona, Spain, April 2006.

[15] H. Zhai and Y. Fang, "Physical carrier sensing and spatial reuse in multirate and multihop wireless ad hoc networks," in Proc. INFOCOM'06, Barcelona, Spain, April 2006.

[16] H. Ma, H. M. K. Alazemi, and S. Roy, "A stochastic model for optimizing physical carrier sensing and spatial reuse in wireless ad hoc networks," MASS 2005.

[17] Y. Zhu, Q. Zhang, Z. Niu, and J. Zhu, "Improve transmission reliability with multi-AP diversity in wireless networks: architecture and performance analysis," in Proc. Third International Conference on Quality of Service in Heterogeneous Wired/Wireless Networks (QShine), Waterloo, Ontario, Canada, Aug. 2006.
[18] J. Padhye, V. Firoiu, D. Towsley, and J. Kurose, "Modeling TCP Reno performance: a simple model and its empirical validation," IEEE/ACM Trans. Networking, vol. 8, pp. 133-145, Apr. 2000.

[19] K. Wang, F. Yang, Q. Zhang, D. Wu, and Y. Xu, "Distributed cooperative rate adaptation for energy efficiency in IEEE 802.11-based multi-hop networks," IEEE Trans. Veh. Technol., vol. 56, no. 2, pp. 888-898, Mar. 2007.

[20] T. S. Rappaport, Wireless Communications: Principles and Practice. Upper Saddle River, NJ: Prentice Hall PTR, 1996.

[21] K. Fall and K. Varadhan, ed., "NS notes and documentation," The VINT Project, UC Berkeley, LBL, USC/ISI, and Xerox PARC, Nov. 1997.

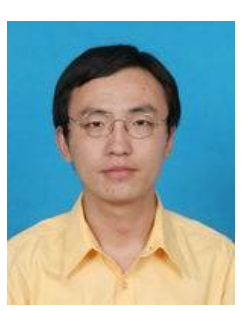

Yanfeng Zhu [S'05] received the B.S. degree in telecommunication from Beijing University of Posts and Telecommunications. He is currently working toward the Ph.D. degree in electronic engineering at the Tsinghua University, China. His research interests include MAC enhancement for IEEE 802.11 WLANs, performance analysis of cooperative communications, and physical carrier sensing and MultiAP cooperation in High-Density wireless networks.

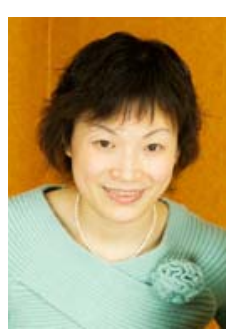

Qian Zhang [M'00, SM'04] (qianzh@cs.ust.hk) is an associate professor in the Department of Computer Science and Engineering, Hong Kong University of Science and Technology. She has published more than 150 refereed papers in international leading journals and key conferences in the areas of wireless/Internet multimedia networking, wireless communications and networking, and overlay networking. She is an Associate Editor for IEEE Transactions on Wireless Communications, IEEE Transactions on Vehicular Technologies, IEEE Transactions on Multimedia, and Computer Networks. She received the TR 100 (MIT Technology Review) world's top young innovator award in 2004.

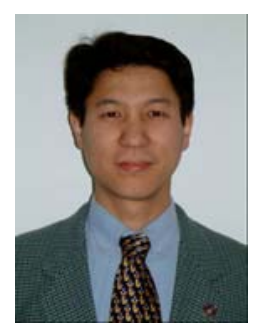

Zhisheng Niu $\left[M^{\prime} \quad 98, \quad\right.$ SM' 99] (niuzhs@tsinghua.edu.cn) graduated from Northern Jiaotong University, Beijing, China, in 1985, and got his M.E. and D.E. degrees from Toyohashi University of Technology, Japan, in 1989 and 1992, respectively. In 1994 he joined Tsinghua University, Beijing, China, where he is now a professor in the Department of Electronic Engineering. From April 1992 to March 1994 he was with Fujitsu Laboratories Ltd., Kawasaki, Japan. His current research interests include teletraffic theory, radio resource management, mobile Internet, and stratospheric communication systems.

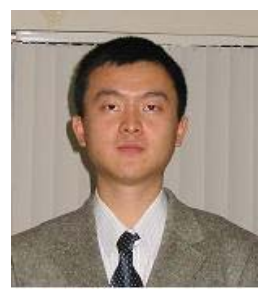

Jing Zhu received his B.S. and M.Sc. degrees both in electronic engineering from Tsinghua University, Beijing, P. R. China, in 2000 and 2001, respectively, and Ph.D. in electrical engineering from University of Washington (Seattle) in 2004. Since 2004, he has been working as a senior research scientist in the Communications Technology Lab at Intel Corp. He is an IEEE member. His main research interests are cross-layer performance optimization for heterogeneous wireless networks, such as land mobile satellite system, next generation cellular system, highdensity wireless LAN, mobile ad-hoc network, etc. and their applications, such as Internet-on-move, digital home/enterprise, intelligent transport system, etc. 